International Mathematical Forum, 2, 2007, no. 42, 2083 - 2097

\title{
Sensitivity Analysis of Units with Interval Data in DEA
}

\author{
Mohammad Izadikhah ${ }^{1}$ \\ Dept. of Math, Arak branch, Islamic Azad University \\ Arak,tel:+98-861-3663041-9, P.O. Box 38135/567, Iran \\ F. Hosseinzadeh Lotfi \\ Dept. of Math, Science and Research Branch \\ Islamic Azad University, Tehran 14515-775, Iran
}

\begin{abstract}
Data Envelopment Analysis (DEA) requires that the data for all inputs and outputs are known exactly. When some outputs and inputs are known decision variables, such as interval data and ordinal data, the DEA models becomes a nonlinear programming problem and is called imprecise DEA (IDEA). When data assume to be interval, decision making units (DMUs) can divide to three classes as follows: (I) efficient in any cases, (II) efficient in maximal sense and inefficient in minimal sense, (III) always inefficient. In this paper, we discuss a new technique for assessing the sensitivity of efficiency and inefficiency classifications in DEA with interval data in above three cases. Also we find radius of stability for all DMUs. Available bank branch data in Iran was used to illustrate the applicability of this approach
\end{abstract}

Mathematics Subject Classification: Operations Research, 90

Keywords: Data envelopment analysis, Interval data, Sensitivity and stability analysis

\section{Introduction}

Data Envelopment Analysis (DEA) is a mathematical programming approach to performance evaluation $[1,2,4]$. DEA requires that the data for all inputs and outputs are known exactly. Recently Cooper et al. addressed the problem of

\footnotetext{
${ }^{1}$ Corresponding author: Mohammad Izadikhah, E-mail:m_izadikhah@yahoo.com
} 
imprecise data in DEA in its general form [3]. When some outputs and inputs are imprecise data such as interval or ordinal data, the DEA model becomes a nonlinear programming problem and is called imprecise DEA (IDEA). In dealing with this data the obtain models usually are nonlinear. To rectify this drawback Cooper et al. [5] proposed some methods to convert the nonlinear model to a linear one. In addition to it one can read Thompson et al. [11]. Recently Lee et al.[10], Zhu [12], Despotis and Smirlis [6] and Jahanshahloo et al. $[7,8,9]$ investigate to IDEA. When evaluate DMUs under interval data, they can classify as follows: $\left(E^{++}\right)$all the DMUs which are efficient with any combination of their inputs and outputs, $\left(E^{+}\right)$the DMUs which are efficient in their maximal status but for some data levels they loss their efficiency and $\left(E^{-}\right)$the DMUs that are inefficient in each case.

In this paper, we present a new technique for assessing the sensitivity of efficiency and inefficiency classification in DEA with interval data. The focus is on the stability of DMUs that have interval data. Having identified efficient and inefficient DMUs in a DEA analysis with interval data, one may want to know how sensitive these identification are to possible variations in data. Also, a modified CCR model is suggested to sensitive the DMUs with interval data. We develop several linear programming formulations for investigating radius of stability for all DMUs with interval data. The possible data perturbations for preserving the DMUs classifications are computed from the optimal values.

The structure of the paper is as follows: In section 2, we review DEA models for dealing with interval data. Then on the basis of these models, in section 3, we propose some models for determining radius of stability of DMUs. In section 4, the sensitivity and stability analysis method to several data sets are introduced. A concluding section summarizes our main results.

\section{Preliminaries}

Suppose we have $n$ DMUs, where each $D M U_{j},(j \in J, J=1, \ldots, n)$ consumes $m$ inputs $X_{j}=\left(x_{1 j}, \ldots, x_{m j}\right)$ to produce $s$ outputs $Y_{j}=\left(y_{1 j}, \ldots, y_{s j}\right)$. We assume $Y_{j} \geq 0, X_{j} \geq 0, Y_{j} \neq 0, X_{j} \neq 0$. Unlike the original DEA model, we assume further that the levels of inputs and outputs are not known exactly, the true input and output data known to lie within bounded intervals i.e. $x_{i j} \in\left[x_{i j}^{L}, x_{i j}^{U}\right]$ and $y_{i j} \in\left[y_{i j}^{L}, y_{i j}^{U}\right]$ with upper and lower bounds of the intervals given as constants and assumed strictly positive. In this case, the efficiency can be an interval. The upper limit of interval efficiency is obtained from the optimistic viewpoint and the lower limit is obtained from the pessimistic 
viewpoint. The following model provides such an upper bound for $D M U_{o}$ :

$$
\begin{aligned}
h_{o}^{U}=\max & \sum_{r=1}^{s} u_{r} y_{r o}^{U} \\
\text { s.t. } & \sum_{i=1}^{m} v_{i} x_{i o}^{L}=1, \\
& \sum_{r=1}^{s} u_{r} y_{r o}^{U}-\sum_{i=1}^{m} v_{i} x_{i o}^{L} \leq 0, \\
& \sum_{r=1}^{s} u_{r} y_{r j}^{L}-\sum_{i=1}^{m} v_{i} x_{i j}^{U} \leq 0, \quad j=1, \ldots, n, j \neq o \\
& u_{r}, v_{i} \geq \varepsilon, \\
& r=1, \ldots, s, i=1, \ldots, m .
\end{aligned}
$$

where $\varepsilon$ is a non-Archimedean infinitesimal and original variables $u_{1}, \ldots, u_{s}$ and $v_{1}, \ldots, v_{m}$ are weights for outputs and inputs, respectively, which to be estimated. We denote by $h_{o}^{U}$ the efficiency score attained by $D M U_{o}$ in (1). The model below provides a lower bound of efficiency score for $D M U_{o}$ :

$$
\begin{aligned}
h_{o}^{L}=\max & \sum_{r=1}^{s} u_{r} y_{r o}^{L} \\
\text { s.t. } & \sum_{i=1}^{m} v_{i} x_{i o}^{U}=1, \\
& \sum_{r=1}^{s} u_{r} y_{r o}^{L}-\sum_{i=1}^{m} v_{i} x_{i o}^{U} \leq 0, \\
& \sum_{r=1}^{s} u_{r} y_{r j}^{U}-\sum_{i=1}^{m} v_{i} x_{i j}^{L} \leq 0, \quad j=1, \ldots, n, j \neq o \\
& u_{r}, v_{i} \geq \varepsilon, \\
& r=1, \ldots, s, i=1, \ldots, m .
\end{aligned}
$$

The efficiency $h_{o}^{L}$ attained by $D M U_{o}$ in (2) serves as a lower bound of its possible efficiency scores. Models (1) and (2) provide each DMU with a bounded interval $\left[h_{o}^{L}, h_{o}^{U}\right]$ in which its possible efficiency scores lie from the worst to the best case. Considering (1) and (2), it is evident that $h_{o}^{L} \leq h_{o}^{U}$. On the basis of the above efficiency score intervals, DMUs can be classified in three subsets as follows:

$$
\begin{aligned}
& E^{++}=\left\{j \in J \mid h_{j}^{L}=1\right\} \\
& E^{+}=\left\{j \in J \mid h_{j}^{L}<1 \text { and } h_{j}^{U}=1\right\} \\
& E^{-}=\left\{j \in J \mid h_{j}^{U}<1\right\}
\end{aligned}
$$

\section{Sensitivity analysis in DEA with interval data}

Suppose that DMUs are evaluated by (1) and (2) are classified in $E^{++}, E^{+}$ and $E^{-}$. Having identified efficient and inefficient DMUs in a DEA analysis, one may want to know how to sensitive these identifications are to possible 
variations in the data. We determine "radius of stability" within which data variations will not alter a DMU's classification from efficient to inefficient status or vice versa. We consider the radius of stability of $D M U_{o}$ in three cases as follow.

\subsection{Radius of stability for DMU belonging to $E^{++}$}

In this case, we assume that $D M U_{o}$ is in $E^{++}$, that is, $h_{o}^{L}=1$. It is obvious that $D M U_{o}$ remains in $E^{++}$if its outputs increase or its inputs decrease. Our aim is to find the scalars $\alpha$ and $\beta$ such that if we decrease upper bound of outputs and increase lower bound of inputs of $D M U_{o}$ by $\alpha$ then $h_{o}^{U}=1$, also if we decrease lower bound of outputs and increase upper bound of inputs of $D M U_{o}$ by $\beta$ then $h_{o}^{L}=1$, i.e. DMU remains in $E^{++}$.

We will calculate the upper-bounds of $\alpha$ and $\beta$, namely the ranges for $\alpha$ and $\beta$, such that the inputs and outputs of $D M U_{o}$ are perturbed within these ranges and the efficiency of $D M U_{o}$ is preserved in $E^{++}$. It has been assumed that $\alpha$ and $\beta$ are scalars and strictly positive. Here, we consider the following cases:

(1) It is obvious that if $X_{o}^{L}-\mathbf{1}_{m} \alpha, Y_{o}^{U}+\mathbf{1}_{s} \alpha$ then $h_{o}^{U}=1$ and if $X_{o}^{U}-\mathbf{1}_{m} \beta$, $Y_{o}^{L}+\mathbf{1}_{s} \beta$ then $h_{o}^{L}=1$; consequently, $D M U_{o} \in E^{++}$(Note that $\mathbf{1}$ is a vector with all components equal 1. ).

(2) If $X_{o}^{L}+\mathbf{1}_{m} \alpha, Y_{o}^{U}-\mathbf{1}_{s} \alpha$ and $X_{o}^{U}+\mathbf{1}_{m} \beta, Y_{o}^{L}-\mathbf{1}_{s} \beta$, then it is possible for $D M U_{o}$ not to be in the $E^{++}$. We are concerned with finding the largest value for $\alpha$ and $\beta$ such that $D \widehat{M U} U_{o} \in E^{++}$, where $D \widehat{M U}_{o}$ is $D M U_{o}$ with the following changes:

$(I):\left\{\begin{array}{c}X_{o}^{L} \rightarrow X_{o}^{L}+\mathbf{1}_{m} \alpha, \\ Y_{o}^{U} \rightarrow Y_{o}^{U}-\mathbf{1}_{s} \alpha,\end{array}\right.$ and

(II): $\left\{\begin{array}{c}X_{o}^{U} \rightarrow X_{o}^{U}+\mathbf{1}_{m} \beta, \\ Y_{o}^{L} \rightarrow Y_{o}^{L}-\mathbf{1}_{s} \beta,\end{array}\right.$

For this purpose, we present the following models:

$$
\begin{array}{lll}
\max & \beta \\
\text { s.t. } & \sum_{r=1}^{s} u_{r}+\sum_{i=1}^{m} v_{i}=1, & \\
& \sum_{r=1}^{s} u_{r}\left(y_{r o}^{L}-\beta\right)-\sum_{i=1}^{m} v_{i}\left(x_{i o}^{U}+\beta\right) \geq 0, & (*) \\
& \sum_{r=1}^{s} u_{r} y_{r j}^{U}-\sum_{i=1}^{m} v_{i} x_{i j}^{L} \leq 0, & j=1, \ldots, n, j \neq o \\
& u_{r}, v_{i} \geq \varepsilon, & r=1, \ldots, s, i=1, \ldots, m .
\end{array}
$$


and

$$
\begin{aligned}
& \max \alpha \\
& \text { s.t. } \sum_{r=1}^{s} u_{r}+\sum_{i=1}^{m} v_{i}=1 \text {, } \\
& \sum_{r=1}^{s} u_{r}\left(y_{\text {ro }}^{U}-\alpha\right)-\sum_{i=1}^{m} v_{i}\left(x_{i o}^{L}+\alpha\right) \geq 0, \quad(* *) \\
& \sum_{r=1}^{s} u_{r} y_{r j}^{U}-\sum_{i=1}^{m} v_{i} x_{i j}^{L} \leq 0, \quad j=1, \ldots, n, j \neq o, \\
& x_{i o}^{L}+\alpha \leq x_{i o}^{U}+\beta^{*}, \quad i=1, \ldots, m, \\
& y_{r o}^{U}-\alpha \geq y_{r o}^{L}-\beta^{*}, \quad r=1, \ldots, s, \\
& u_{r}, v_{i} \geq \varepsilon \text {, }
\end{aligned}
$$

Where, $\beta^{*}$ is optimal value of model (3). In models (3) and (4), inequality in constrains $(*)$ and $(* *)$ guarantees that these models are feasible. Here, the constraint $\sum_{r=1}^{s} u_{r}+\sum_{i=1}^{m} v_{i}=1$ is added for normalization purpose. In the first glance, it seems that (3) and (4) are nonlinear, but the following manipulation transforms the models into linear.

The constraint $\left(^{*}\right)$ converted to $\sum_{r=1}^{s} u_{r} y_{r o}^{L}-\sum_{i=1}^{m} v_{i} x_{i o}^{U} \geq \beta$. Similarly, constraint $(* *)$ converted to $\sum_{r=1}^{s} u_{r} y_{r o}^{L}-\sum_{i=1}^{m} v_{i} x_{i o}^{U} \geq \alpha$.

By the above substitutions, the following linear models are obtained:

$$
\begin{array}{ll}
\max & \beta \\
\text { s.t. } & \sum_{r=1}^{s} u_{r}+\sum_{i=1}^{m} v_{i}=1, \\
& \sum_{r=1}^{s} u_{r} y_{r o}^{L}-\sum_{i=1}^{m} v_{i} x_{i o}^{U} \geq \beta \\
& \sum_{r=1}^{s} u_{r} y_{r j}^{U}-\sum_{i=1}^{m} v_{i} x_{i j}^{L} \leq 0, \quad j=1, \ldots, n, j \neq o \\
& u_{r}, v_{i} \geq \varepsilon, \\
& r=1, \ldots, s, i=1, \ldots, m .
\end{array}
$$


and

$$
\begin{aligned}
& \max \alpha \\
& \text { s.t. } \sum_{r=1}^{s} u_{r}+\sum_{i=1}^{m} v_{i}=1 \text {, } \\
& \sum_{r=1}^{s} u_{r} y_{r o}^{L}-\sum_{i=1}^{m} v_{i} x_{i o}^{U} \geq \alpha \\
& \sum_{r=1}^{s} u_{r} y_{r j}^{U}-\sum_{i=1}^{m} v_{i} x_{i j}^{L} \leq 0, \quad j=1, \ldots, n, j \neq o \text {, } \\
& x_{i o}^{L}+\alpha \leq x_{i o}^{U}+\beta^{*}, \quad i=1, \ldots, m, \\
& y_{r o}^{U}-\alpha \geq y_{\text {ro }}^{L}-\beta^{*}, \quad r=1, \ldots, s, \\
& u_{r}, v_{i} \geq \varepsilon, \quad r=1, \ldots, s, i=1, \ldots, m \text {. }
\end{aligned}
$$

Where, $\beta^{*}$ is optimal value of model (5).

Theorem 1. After solving models (5) and (6) and applying the changes (I) and (II), the data of $D M U_{o}$ remain interval.

Proof. With regard to following constrains,

$$
\begin{gathered}
X_{i o}^{L}+\alpha \leq X_{i o}^{U}+\beta, \quad i=1, \ldots, m \\
Y_{r o}^{U}-\alpha \geq Y_{r o}^{L}-\beta^{*}, \quad r=1, \ldots, s .
\end{gathered}
$$

proof is evident.

Theorem 2. Suppose $D M U_{o}$ is in $E^{++}$. After solving models (5) and (6) and applying the changes (I) and (II), $D M U_{o}$ remains a member of $E^{++}$.

Proof. In theorem 1 we showed that the data of $\widehat{D M U} U_{o}$ are interval. (Where $D \widehat{M U}_{o}$ is $D M U_{o}$ after changes (I) and (II)). To showing that $D \widehat{M U}_{o}^{++}$, it is necessary to prove $h_{o}^{L}=1$. Where

$$
\begin{aligned}
& h_{o}^{L}=\max \sum_{r=1}^{s} u_{r}\left(y_{r o}^{L}-\beta^{*}\right) \\
& \text { s.t. } \sum_{i=1}^{m} v_{i}\left(x_{i o}^{U}+\beta^{*}\right)=1 \text {, } \\
& \sum_{r=1}^{s} u_{r}\left(y_{r o}^{L}-\beta^{*}\right)-\sum_{i=1}^{m} v_{i}\left(x_{i o}^{U}+\beta^{*}\right) \leq 0 \\
& \sum_{r=1}^{s} u_{r} y_{r j}^{U}-\sum_{i=1}^{m} v_{i} x_{i j}^{L} \leq 0, \quad j=1, \ldots, n, j \neq 0, \\
& u_{r}, v_{i} \geq \varepsilon, \quad r=1, \ldots, s, i=1, \ldots, m \text {. }
\end{aligned}
$$

where, $\beta^{*}$ is optimal value of model (3). Suppose $\left(u^{*}, v^{*}, \beta^{*}\right)$ be the optimal solution of model (3), then we have:

$$
\begin{array}{ll}
\sum_{r=1}^{s} u_{r}^{*}\left(y_{r o}^{L}-\beta^{*}\right)-\sum_{i=1}^{m} v_{i}^{*}\left(x_{i o}^{U}+\beta^{*}\right) \geq 0, & \\
\sum_{r=1}^{s} u_{r}^{*} y_{r j}^{U}-\sum_{i=1}^{m} v_{i}^{*} x_{i j}^{L} \leq 0, & j=1, \ldots, n, j \neq 0 .
\end{array}
$$


we define $\gamma$ as follows: $\gamma=\sum_{i=1}^{m} v_{i}^{*}\left(x_{i o}^{U}+\beta^{*}\right) \leq \sum_{r=1}^{s} u_{r}^{*}\left(y_{r o}^{L}-\beta^{*}\right)$, (clearly, $\left.\gamma>0\right)$. And by following substitutions:

$$
\begin{aligned}
& \overline{u_{r}}=\frac{u_{r}^{*}}{\gamma}>0, r=1, \ldots, s, \\
& \overline{v_{i}}=\frac{v_{i}^{*}}{\gamma}>0, i=1, \ldots, m .
\end{aligned}
$$

We have, $\sum_{i=1}^{m} \bar{v}_{i}\left(x_{i o}^{U}+\beta^{*}\right)=1$ and therefore, $\sum_{r=1}^{s} u_{r}^{*}\left(y_{\text {ro }}^{L}-\beta^{*}\right)=1$. Therefore $(\bar{u}, \bar{v})$ is a feasible solution for model (7) with objective function $\sum_{r=1}^{s} u_{r}^{*}\left(y_{r o}^{L}-\beta^{*}\right)=1$. Then $(\bar{u}, \bar{v})$ is optimal solution of model $(7)$ and we have $h_{o}^{L}=1$.

\subsection{Radius of stability for DMU belonging to $\mathrm{E}^{+}$}

In this case, we assume that $D M U_{o}$ is in $E^{+}$, that is, $h_{o}^{L}<0$ and $h_{o}^{U}=1$. Our aim is to find the scalars $\alpha$ and $\beta$ such that, if we decrease upper bound of outputs and increase lower bound of inputs of $D M U_{o}$ by $\alpha$ then $h_{o}^{U}=1$, also if we increase lower bound of outputs and decrease upper bound of inputs of $D M U_{o}$ by $\beta$ then $h_{o}^{L}<1$, i.e. $D M U_{o}$ remains in $E^{+}$. We will calculate the upper bounds of $\alpha$ and $\beta$, namely the ranges for alpha and $\beta$ such that the inputs and outputs of $D M U_{o}$ perturbations within these ranges preserve the efficiency of $D M U_{o}$ in $E^{+}$. Here, we consider the following cases:

(1) If $X_{o}^{L} \rightarrow X_{o}^{L}-\mathbf{1}_{m} \alpha, Y_{o}^{U} \rightarrow Y_{o}^{U}+\mathbf{1}_{s} \alpha$ then $h_{o}^{U}=1$ and if $X_{o}^{U}, Y_{o}^{L}$ are fixed, then $h_{o}^{L}<1$, consequently $D M U_{o} \in E^{+}$.

(2) If $X_{o}^{U} \rightarrow X_{o}^{U}+\mathbf{1}_{m} \beta, Y_{o}^{L} \rightarrow Y_{o}^{L}-\mathbf{1}_{s} \alpha$ then $h_{o}^{L}<1$ and if $X_{o}^{L}, Y_{o}^{U}$ are fixed, then $h_{o}^{U}=1$, consequently $D M U_{o} \in E^{+}$.

(3) We are concerned in finding the largest values of $\alpha$ and $\beta$ such that $D \widehat{M U}_{o}^{+}$, where $D \widehat{M U}_{o}$ is $D M U_{o}$ with the following changes:

$$
(I I I):\left\{\begin{array}{c}
X_{o}^{L} \rightarrow X_{o}^{L}+\mathbf{1}_{m} \alpha \\
Y_{o}^{U} \rightarrow Y_{o}^{U}-\mathbf{1}_{s} \alpha
\end{array}\right.
$$

and

$$
(I V):\left\{\begin{array}{c}
X_{o}^{U} \rightarrow X_{o}^{U}-\mathbf{1}_{m} \beta \\
Y_{o}^{L} \rightarrow Y_{o}^{L}+\mathbf{1}_{s} \beta
\end{array}\right.
$$


For this purpose, we present the following models:

$$
\begin{aligned}
& \max \alpha \\
& \text { s.t. } \sum_{r=1}^{s} u_{r}+\sum_{i=1}^{m} v_{i}=1 \text {, } \\
& \sum_{r=1}^{s} u_{r}\left(y_{r o}^{U}-\alpha\right)-\sum_{i=1}^{m} v_{i}\left(x_{i o}^{L}+\alpha\right) \geq 0, \quad(*) \\
& \sum_{r=1}^{s} u_{r} y_{r j}^{L}-\sum_{i=1}^{m} v_{i} x_{i j}^{U} \leq 0, \quad j=1, \ldots, n, j \neq o \text {, } \\
& u_{r}, v_{i} \geq \varepsilon, \quad r=1, \ldots, s, i=1, \ldots, m \text {. }
\end{aligned}
$$

Assume, $\alpha^{*}$ is optimal value of objective function. Then we choose $\bar{\alpha}$ such that $0 \leq \bar{\alpha}<\alpha^{*}$, value of $\bar{\alpha}$ is determined by manager, and then solve the following model:

$$
\begin{array}{lll}
\max & \beta \\
\text { s.t. } & \sum_{r=1}^{s} u_{r}+\sum_{i=1}^{m} v_{i}=1, & \\
& \sum_{r=1}^{s} u_{r}\left(y_{r o}^{L}+\beta\right)-\sum_{i=1}^{m} v_{i}\left(x_{i o}^{U}-\beta\right) \leq 0, & (* *) \\
& \sum_{r=1}^{s} u_{r} y_{r j}^{U}-\sum_{i=1}^{m} v_{i} x_{i j}^{L} \leq 0, & \\
& x_{i o}^{U}-\beta \geq x_{i o}^{L}+\bar{\alpha}, & \\
& y_{r o}^{L}+\beta \leq y_{r o}^{U}-\bar{\alpha}, & r=1, \ldots, m, j \neq o, \\
& u_{r}, v_{i} \geq \varepsilon, & r=1, \ldots, s, \\
& &
\end{array}
$$

In models (8) and (9), inequality in constrains $(*)$ and $(* *)$ guarantees that these models are feasible. Clearly, models (8) and (9) are nonlinear, but by similar manipulation as mentioned in section (3.1) transform in to following linear models:

$$
\begin{array}{ll}
\max & \alpha \\
\text { s.t. } & \sum_{r=1}^{s} u_{r}+\sum_{i=1}^{m} v_{i}=1, \\
& \sum_{r=1}^{s} u_{r} y_{r o}^{U}-\sum_{i=1}^{m} v_{i} x_{i o}^{L} \geq \alpha, \\
& \sum_{r=1}^{s} u_{r} y_{r j}^{L}-\sum_{i=1}^{m} v_{i} x_{i j}^{U} \leq 0, \quad j=1, \ldots, n, j \neq o, \\
& u_{r}, v_{i} \geq \varepsilon, \\
& r=1, \ldots, s, i=1, \ldots, m .
\end{array}
$$


and

$$
\begin{aligned}
& \max \beta \\
& \text { s.t. } \sum_{r=1}^{s} u_{r}+\sum_{i=1}^{m} v_{i}=1 \text {, } \\
& \sum_{r=1}^{s} u_{r} y_{r o}^{L}-\sum_{i=1}^{m} v_{i} x_{i o}^{U}+\beta \leq 0 \text {, } \\
& \sum_{r=1}^{s} u_{r} y_{r j}^{U}-\sum_{i=1}^{m} v_{i} x_{i j}^{L} \leq 0, \quad j=1, \ldots, n, j \neq o \\
& \stackrel{r=1}{U}_{i o}^{U}-\beta \geq x_{i o}^{i=1}+\bar{\alpha}, \quad i=1, \ldots, m, \\
& y_{\text {ro }}^{L}+\beta \leq y_{\text {ro }}^{U}-\bar{\alpha}, \quad r=1, \ldots, s, \\
& u_{r}, v_{i} \geq \varepsilon \text {, } \\
& r=1, \ldots, s, i=1, \ldots, m \text {. }
\end{aligned}
$$

Where $0 \leq \bar{\alpha}<\alpha^{*}$.

Theorem 3. After solving models (10) and (11) and applying the changes (III) and (IV), the data of $D M U_{o}$ remain interval.

Proof. proof is evident.

Theorem 4. Suppose $D M U_{o}$ is in $E^{+}$. Then $D \widehat{M U} U_{o}$ with inputs $x_{i o}$ where $x_{i o}^{L}+\bar{\alpha}_{i o} \leq x_{i o}^{U}-\beta^{*}$, and outputs $y_{\text {ro }}$ where $y_{r o}^{L}+\beta_{r o}^{*} \leq y_{r o}^{U}-\bar{\alpha}$ be a member of $E^{+}$.

Proof. The proof is similar to that of theorem (2) and is omitted.

\subsection{Radius of stability for DMU belonging to $\mathrm{E}^{-}$}

In this case, we assume that $D M U_{o}$ is in $E^{-}$. Our aim is to find the scalars $\alpha$ and $\beta$ such that if we decrease lower bound of inputs and increase upper bound of outputs of $D M U_{o}$ by $\alpha$ then $h_{o}^{U}<1$, also if we decrease upper bound of inputs and increase lower bound of outputs of $D M U_{o}$ by $\beta$ then $h_{o}^{L}<1$, i.e. $D M U_{o}$ remains in $E^{-}$. We will calculate the upper bounds of $\alpha$ and $\beta$, namely the ranges for $\alpha$ and $\beta$ such that the inputs and outputs of $D M U_{o}$ are perturbed within these ranges and its inefficiency score is not changed. Now we consider following cases:

(1) If $X_{o}^{L} \rightarrow X_{o}^{L}+\mathbf{1}_{m} \alpha$ and $Y_{o}^{U} \rightarrow Y_{o}^{U}-\mathbf{1}_{s} \alpha$ then $h_{o}^{U}<1$ and if $X_{o}^{U} \rightarrow$ $X_{o}^{U}+\mathbf{1}_{m} \beta$ and $Y_{o}^{L} \rightarrow Y_{o}^{L}-\mathbf{1}_{s} \beta$ then $h_{o}^{L}<1$, consequently $D M U_{o} \in E^{-}$.

(2) If $X_{o}^{L} \rightarrow X_{o}^{L}-\mathbf{1}_{m} \alpha, Y_{o}^{U} \rightarrow Y_{o}^{U}+\mathbf{1}_{s} \alpha$ and if $X_{o}^{U} \rightarrow X_{o}^{U}-\mathbf{1}_{m} \beta, Y_{o}^{L} \rightarrow$

$Y_{o}^{L}+\mathbf{1}_{s} \beta$ then it is possible for $D M U_{o}$ not to be in the $E^{-}$. We are concerned with finding the largest value for $\alpha$ and $\beta$ such that $D \widehat{M U}_{o}{ }^{-}$, where $D \widehat{M U} U_{o}$ is $D M U_{o}$ with the following changes:

$$
(V):\left\{\begin{array}{c}
X_{o}^{L} \rightarrow X_{o}^{L}-\mathbf{1}_{m} \alpha \\
Y_{o}^{U} \rightarrow Y_{o}^{U}+\mathbf{1}_{s} \alpha
\end{array}\right.
$$

and 


$$
(V I):\left\{\begin{array}{c}
X_{o}^{U} \rightarrow X_{o}^{U}-\mathbf{1}_{m} \beta \\
Y_{o}^{L} \rightarrow Y_{o}^{L}+\mathbf{1}_{s} \beta
\end{array}\right.
$$

For this purpose, we present the following models:

$$
\begin{aligned}
& \min \alpha \\
& \text { s.t. } \sum_{r=1}^{s} u_{r}+\sum_{i=1}^{m} v_{i}=1 \text {, } \\
& \sum_{r=1}^{s} u_{r}\left(y_{r_{o}}^{U}+\alpha\right)-\sum_{i=1}^{m} v_{i}\left(x_{i o}^{L}-\alpha\right) \geq 0, \\
& \sum_{r=1}^{s} u_{r} y_{r j}^{L}-\sum_{i=1}^{m} v_{i} x_{i j}^{U} \leq 0 \\
& j=1, \ldots, n, j \neq o, \\
& u_{r}, v_{i} \geq \varepsilon \text {, } \\
& r=1, \ldots, s, i=1, \ldots, m \text {. }
\end{aligned}
$$

Assume, $\alpha^{*}$ is optimal value of objective function. Since, we must $h_{o}^{L}<1$, so we choose $\bar{\alpha}$ such that $0 \leq \bar{\alpha}<\alpha^{*}$, value of $\bar{\alpha}$ is determined by manager, then solve the following model:

$$
\begin{array}{lll}
\min & \beta \\
\text { s.t. } & \sum_{r=1}^{s} u_{r}+\sum_{i=1}^{m} v_{i}=1, & \\
& \sum_{r=1}^{s} u_{r}\left(y_{r o}^{L}+\beta\right)-\sum_{i=1}^{m} v_{i}\left(x_{i o}^{U}-\beta\right) \geq 0, & (* *) \\
& \sum_{r=1}^{s} u_{r} y_{r j}^{L}-\sum_{i=1}^{m} v_{i} x_{i j}^{U} \leq 0, & j=1, \ldots, n, j \neq o, \\
& x_{i o}^{U}-\beta \geq x_{i o}^{L}-\bar{\alpha}, & \\
y_{r o}^{L}+\beta \leq y_{r o}^{U}+\bar{\alpha}, & r=1, \ldots, m, \\
& u_{r}, v_{i} \geq \varepsilon, & r=1, \ldots, s, i=1, \ldots, m .
\end{array}
$$

In models (12) and (13), inequality in constrains $\left(^{*}\right)$ and $(* *)$ guarantees that these models are feasible. Clearly, models (12) and (13) are nonlinear, so by similar manipulation as mentioned in section (3.1) transform in to following linear models:

$$
\begin{aligned}
& \min \alpha \\
& \text { s.t. } \sum_{r=1}^{s} u_{r}+\sum_{i=1}^{m} v_{i}=1 \text {, } \\
& \sum_{r=1}^{s} u_{r} y_{r o}^{U}-\sum_{i=1}^{m} v_{i} x_{i o}^{L}+\alpha \geq 0 \\
& \sum_{r=1}^{r=1} u_{r} y_{r j}^{L}-\sum_{i=1}^{m} v_{i} x_{i j}^{U} \leq 0, \quad j=1, \ldots, n, j \neq o, \\
& u_{r}, v_{i} \geq \varepsilon, \quad r=1, \ldots, s, i=1, \ldots, m \text {. }
\end{aligned}
$$


and

$$
\begin{array}{lll}
\min & \beta \\
\text { s.t. } & \sum_{r=1}^{s} u_{r}+\sum_{i=1}^{m} v_{i}=1, & \\
& \sum_{r=1}^{s} u_{r} y_{r o}^{L}-\sum_{i=1}^{m} v_{i} x_{i o}^{U}+\beta \geq 0, & \\
& \sum_{r=1}^{s} u_{r} y_{r j}^{L}-\sum_{i=1}^{m} v_{i} x_{i j}^{U} \leq 0, & j=1, \ldots, n, j \neq o, \\
& x_{i o}^{U}-\beta \geq x_{i o}^{L}-\bar{\alpha}, & i=1, \ldots, m, \\
& y_{r o}^{L}+\beta \leq y_{r o}^{U}+\bar{\alpha}, & r=1, \ldots, s, \\
& u_{r}, v_{i} \geq \varepsilon, & r=1, \ldots, s, i=1, \ldots, m .
\end{array}
$$

Theorem 5. After solving models (14) and (15) and applying the changes $(\mathrm{V})$ and $(\mathrm{VI})$, the data of $D M U_{o}$ remain interval.

Proof. Proof is evident.

Theorem 6. Suppose $D M U_{o}$ is in $E^{-}$. Then $D \widehat{M U} U_{o}$ with inputs $x_{i o}$ where $x_{i o}^{L}-\bar{\alpha} \leq x_{i o} \leq x_{i o}^{U}-\beta^{*}$, and outputs $y_{\text {ro }}$ where $y_{r o}^{L}+\beta^{*} \leq y_{\text {ro }} \leq y_{\text {ro }}^{U}+\bar{\alpha}$ is also a member of $E^{-}$.

Proof. The proof is similar to that of theorem 2 and is omitted.

\section{An application in Bank Branches in Iran}

We now apply this approach to some commercial bank branches in Iran. There are 20 branches in this district. Each branch uses 3 inputs to produce 5 outputs. Table 1 shows the kind of these inputs and outputs.

Table 1.

Table 1.
\begin{tabular}{|c|c|}
\hline Inputs & Outputs \\
\hline Payable interest & The total sum of four main deposits \\
Personnel & Other deposits \\
Non-Performing loans & Loans granted \\
& Received interest \\
& Fee \\
\hline
\end{tabular}

In Tables 2 and 3 the interval inputs and interval outputs for these DMUs are given. 
Table 2.Input-data for the 20 bank branches

\begin{tabular}{|ccccccc|}
\hline$D M U_{j}$ & $x_{1 j}^{L}$ & $x_{1 j}^{U}$ & $x_{2 j}^{L}$ & $x_{2 j}^{U}$ & $x_{3 j}^{L}$ & $x_{3 j}^{U}$ \\
\hline 1 & 5007.37 & 9613.37 & 36.29 & 36.86 & 87243 & 87243 \\
2 & 2926.81 & 5961.55 & 18.8 & 2016 & 9945 & 12120 \\
3 & 8732.7 & 17752.5 & 25.74 & 27.17 & 47575 & 50013 \\
4 & 945.93 & 1966.39 & 20.81 & 22.54 & 19292 & 19753 \\
5 & 8487.07 & 17521.66 & 14.16 & 14.8 & 3428 & 3911 \\
6 & 13759.35 & 27359.36 & 19.46 & 19.46 & 13929 & 15657 \\
7 & 587.69 & 1205.47 & 27.29 & 27.48 & 27827 & 29005 \\
8 & 4646.39 & 9559.61 & 24.52 & 25.07 & 9070 & 9983 \\
9 & 1554.29 & 3427.89 & 20.47 & 21.59 & 412036 & 413902 \\
10 & 17528.31 & 36297.54 & 14.84 & 15.05 & 8638 & 10229 \\
11 & 2444.34 & 4955.78 & 20.42 & 20.54 & 500 & 937 \\
12 & 7303.27 & 14178.11 & 22.87 & 23.19 & 16148 & 21353 \\
13 & 9852.15 & 19742.89 & 18.47 & 21.83 & 17163 & 17290 \\
14 & 4540.75 & 9312.24 & 22.83 & 23.96 & 17918 & 17964 \\
15 & 3039.58 & 6304.01 & 39.32 & 39.86 & 51582 & 55136 \\
16 & 6585.81 & 13453.58 & 25.57 & 26.52 & 20975 & 23992 \\
17 & 4209.18 & 8603.79 & 27.59 & 27.95 & 41960 & 43103 \\
18 & 1015.52 & 2037.82 & 13.63 & 13.93 & 18641 & 19354 \\
19 & 5800.38 & 11875.39 & 27.12 & 27.26 & 19500 & 19569 \\
20 & 1445.68 & 2922.15 & 28.96 & 28.96 & 31700 & 32061 \\
\hline
\end{tabular}

Table 3. Output-data for the 20 bank branches

\begin{tabular}{|c|c|c|c|c|c|c|c|c|c|c|}
\hline$D M U_{j}$ & $y_{1 j}^{L}$ & $y_{1 j}^{U}$ & $y_{2 j}^{L}$ & $y_{2 j}^{U}$ & $y_{3 j}^{L}$ & $y_{3 j}^{U}$ & $y_{4 j}^{L}$ & $y_{4 j}^{U}$ & $y_{5 j}^{L}$ & $y_{5 j}^{U}$ \\
\hline 1 & 2696995 & 3126798 & 263643 & 382545 & 1675519 & 1853365 & 108634.76 & 125740.28 & 965.97 & 6957.33 \\
\hline 2 & 340377 & 440355 & 95978 & 117659 & 377309 & 390203 & 32396.65 & 37836.56 & 304.67 & 749.4 \\
\hline 3 & 1027546 & 1061260 & 37911 & 503089 & 1233548 & 1822028 & 96842.33 & 108080.01 & 2285.03 & 3174 \\
\hline 4 & 1145235 & 1213541 & 229646 & 268460 & 468520 & 542101 & 32362.8 & 39273.37 & 207.98 & 510.93 \\
\hline 5 & 390902 & 395241 & 4924 & 12136 & 129751 & 142873 & 12662.71 & 14165.44 & 63.32 & 92.3 \\
\hline 6 & 988115 & 1087392 & 74133 & 111324 & 507502 & 574355 & 53591.3 & 72257.28 & 480.16 & 869.52 \\
\hline 7 & 144906 & 165818 & 180530 & 180617 & 288513 & 323721 & 40507.97 & 45847.48 & 176.58 & 370.81 \\
\hline 8 & 408163 & 416416 & 405396 & 486431 & 1044221 & 1071812 & 56260.09 & 73948.09 & 4654.71 & 5882.53 \\
\hline 9 & 335070 & 410427 & 337971 & 449336 & 1584722 & 1802942 & 176436.81 & 189006.12 & 560.26 & 2506.67 \\
\hline 10 & 700842 & 768593 & 14378 & 15192 & 2290745 & 2573512 & 662725.21 & 791463.08 & 58.89 & 86.86 \\
\hline 11 & 641680 & 696338 & 114183 & 241081 & 1579961 & 2285079 & 17527.58 & 20773.91 & 1070.81 & 2283.08 \\
\hline 12 & 453170 & 481943 & 27196 & 29553 & 245726 & 275717 & 35757.83 & 42790.14 & 375.07 & 559.85 \\
\hline 13 & 553167 & 574989 & 21298 & 23043 & 425886 & 431815 & 45652.24 & 50255.75 & 438.43 & 836.82 \\
\hline 14 & 309670 & 342598 & 20168 & 26172 & 124188 & 126930 & 8143.79 & 11948.04 & 936.62 & 1468.45 \\
\hline 15 & 286149 & 317186 & 149183 & 270708 & 787959 & 810088 & 106798.63 & 111962.3 & 1203.79 & 4335.24 \\
\hline 16 & 321435 & 347848 & 66169 & 80453 & 360880 & 379488 & 89971.47 & 165524.22 & 200.36 & 399.8 \\
\hline 17 & 618105 & 835839 & 244250 & 404579 & 9136507 & 9136507 & 33036.79 & 41826.51 & 2781.24 & 4555.42 \\
\hline 18 & 248125 & 320974 & 3063 & 6330 & 26687 & 29173 & 9525.6 & 10877.78 & 240.04 & 274.7 \\
\hline 19 & 640890 & 679916 & 490508 & 684372 & 2946797 & 3985900 & 66097.16 & 95329.87 & 961.56 & 1914.25 \\
\hline 20 & 119948 & 120208 & 14943 & 17495 & 297674 & 308012 & 21991.53 & 27934.19 & 282.73 & 471.22 \\
\hline
\end{tabular}

Table 4. Efficiency units and classification

\begin{tabular}{|cccc|cccc|}
\hline$D M U_{j}$ & $h_{j}^{U}$ & $h_{j}^{L}$ & Classification & $D M U_{j}$ & $h_{j}^{U}$ & $h_{j}^{L}$ & Classification \\
\hline 1 & 1.000 & 1.000 & $E^{++}$ & 11 & 1.000 & 1.000 & $E^{++}$ \\
2 & 1.000 & 0.371 & $E^{+}$ & 12 & 0.496 & 0.328 & $E^{-}$ \\
3 & 1.000 & 0.523 & $E^{+}$ & 13 & 0.701 & 0.449 & $E^{-}$ \\
4 & 1.000 & 1.000 & $E^{++}$ & 14 & 0.724 & 0.263 & $E^{-}$ \\
5 & 0.763 & 0.618 & $E^{-}$ & 15 & 1.000 & 0.413 & $E^{+}$ \\
6 & 1.000 & 0.917 & $E^{+}$ & 16 & 1.000 & 0.221 & $E^{+}$ \\
7 & 1.000 & 0.728 & $E^{+}$ & 17 & 1.000 & 1.000 & $E^{++}$ \\
8 & 1.000 & 1.000 & $E^{++}$ & 18 & 0.952 & 0.263 & $E^{-}$ \\
9 & 1.000 & 1.000 & $E^{++}$ & 19 & 1.000 & 0.991 & $E^{+}$ \\
10 & 1.000 & 1.000 & $E^{++}$ & 20 & 1.000 & 0.183 & $E^{+}$ \\
\hline
\end{tabular}

In Table 5 we present the radius of stability for DMUs which is belong to set $E^{++}$, that is values of $\beta_{j}^{*}$ and $\alpha_{j}^{*}, \forall j, j \in E^{++}$.

Table 5. Radius of stability for $E^{++}$ 


\begin{tabular}{|ccc|}
\hline$D M U_{j}$ & $\beta_{j}^{*}$ & $\alpha_{j}^{*}$ \\
\hline 1 & 9.733 & 9.733 \\
4 & $6.746 \mathrm{E}+2$ & $6.763 \mathrm{E}+2$ \\
8 & 13.794 & 14.344 \\
9 & 28.107 & 29.227 \\
10 & $2.429 \mathrm{E}+4$ & $2.429 \mathrm{E}+4$ \\
11 & $7.006 \mathrm{E}+3$ & $7.006 \mathrm{E}+3$ \\
17 & $1.129 \mathrm{E}+3$ & $1.129 \mathrm{E}+3$ \\
\hline
\end{tabular}

In Table 6 we present the radius of stability for DMUs which is belong to sets $E^{+}$and $E^{-}$.

Table 6. Radius of stability for DMUs belong to $E^{+}$and $E^{-}$

\begin{tabular}{|cccc|cccc|}
\hline$D M U_{j} \in E^{+}$ & $\alpha_{j}^{*}$ & $\beta_{j}^{*}$ & $\bar{\alpha}$ & $D M U_{j} \in E^{-}$ & $\alpha_{j}^{*}$ & $\beta_{j}^{*}$ & $\bar{\alpha}$ \\
\hline 2 & 29.27 & $4.3 \mathrm{E}+2$ & 14.63 & 5 & 3.95 & 2.61 & 1.97 \\
3 & $2.22 \mathrm{E}+2$ & $-1.09 \mathrm{E}+2$ & $1.11 \mathrm{E}+2$ & 12 & 14.35 & 7.49 & 7.17 \\
6 & 6.50 & -3.25 & 3.25 & 13 & 7.45 & 7.08 & 3.72 \\
7 & $1.74 \mathrm{E}+3$ & $-8.70 \mathrm{E}+2$ & $8.70 \mathrm{E}+2$ & 14 & 12.28 & 7.27 & 6.14 \\
15 & $2.81 \mathrm{E}+3$ & $-1.40 \mathrm{E}+3$ & $1.40 \mathrm{E}+3$ & 18 & 6.60 & 3.60 & 3.30 \\
16 & $1.74 \mathrm{E}+3$ & $-8.73 \mathrm{E}+2$ & $8.74 \mathrm{E}+2$ & & & & \\
19 & $1.01 \mathrm{E}+4$ & $-5.04 \mathrm{E}+3$ & $5.04 \mathrm{E}+3$ & & & & \\
\hline
\end{tabular}

\section{Discussion}

From results of tables $(5,6)$ manager can decided that which data perturbations for preserving the DMUs classifications are suitable. For instance, we can see that, by applying the changes (I),(II),(III),(IV),(V) and (VI) inputs and outputs of $D \widehat{M U}_{9} \in E^{++}$and $\widehat{D M U} \widehat{U}_{6} \in E^{+}$and $D \widehat{M U}_{14} \in E^{-}$are as tables 7 and 8:

Table 7.Input-data

\begin{tabular}{|ccccccc|}
\hline$D M U_{j}$ & $x_{1 j}^{L}$ & $x_{1 j}^{U}$ & $x_{2 j}^{L}$ & $x_{2 j}^{U}$ & $x_{3 j}^{L}$ & $x_{3 j}^{U}$ \\
\hline 9 & 1583.51 & 3455.99 & 49.69 & 49.69 & 412065.22 & 413930.10 \\
6 & 13762.60 & 27362.61 & 22.71 & 22.71 & 13932.25 & 15660.25 \\
14 & 4534.61 & 9304.96 & 16.69 & 16.69 & 17911.86 & 17956.72 \\
\hline
\end{tabular}

Table 8. Output-data

\begin{tabular}{ccccccc}
\hline$D M U_{j}$ & $y_{1 j}^{L}$ & $y_{1 j}^{U}$ & $y_{2 j}^{L}$ & $y_{2 j}^{U}$ & $y_{3 j}^{L}$ & $y_{3 j}^{U}$ \\
\hline 9 & 335041.89 & 410397.77 & 337942.42 & 449306.77 & 1584693.89 & 1802912.77 \\
6 & 988111.74 & 1087388.74 & 74129.74 & 111320.74 & 507498.74 & 574351.74 \\
14 & 309677.27 & 342604.14 & 20175.27 & 26178.14 & 124195.27 & 126936.14 \\
& & & & & & \\
\hline \hline$D M U_{j}$ & $y_{4 j}^{L}$ & $y_{4 j}^{U}$ & $y_{5 j}^{L}$ & $y_{5 j}^{U}$ & & \\
\hline 9 & 176408.70 & 188976.89 & 532.15 & 2477.44 & & \\
6 & 53588.04 & 72254.02 & 476.90 & 856.26 & & \\
14 & 8151.06 & 11954.18 & 943.79 & 1474.59 & & \\
\hline
\end{tabular}

Where, we consider that, in models (10),(11),(14) and (15) manager chooses $\bar{\alpha}=\frac{\alpha^{*}}{2}$. Clearly, by Tables 7 and $8 \widehat{D M U}, \widehat{M U}_{6}$ and $\widehat{D U U}_{14}$ have interval data. 


\section{Conclusion}

The current paper develops a new approach for the sensitivity analysis of DMUs with interval data in three following cases: (I) efficient in any cases, (II) efficient in maximal sense and inefficient in minimal sense, (III) always inefficient. And also, finds the radius of stability and largest region for preserving classification of each DMU. Available bank branch data in Iran was used to illustrate the applicability of this approach.

\section{References}

[1] R. D. Banker, A. Charnes, W. W. Cooper, Some model for estimating technical and scale inefficiencies in data envelopment analysis, Manage. Sci., 30 (1984) 1078-1092.

[2] A. Charnes, W. W. Cooper, E. Rhodes, Measuring the efficiency of decision making unit, European J. Oper. Res., 2 (1978) 429-444.

[3] W. W. Cooper, K. S. Park, G. Yu, IDEA and AR-IDEA: models for dealing with imprecise data in DEA, Manage. Sci., 45 (1999) 597-607.

[4] W. W. Cooper, L. M. Seiford, K. Tone, (2000), Data envelopment analysis ? a comprehensive text with models, applications, references and DEAsolver software. Dordrecht: Kluwer Academic Publisher.

[5] W. W. Copper, K. S. Park, G. Yu, IDEA (Imprecise data envelopment analysis ) with CMDs (Column maximum decision making units), J. Oper. Res. Soc., 52 (2001) 176-181.

[6] D. K. Despotis, Y. G. Smirlis, Data envelopment analysis with imprecise data, European J. Oper. Res., 140 (2002) 24-36.

[7] G. R. Jahanshahloo, F. Hosseinzadeh Lotfi, M. Izadikhah, An algorithmic method to extend TOPSIS for decision-making problems with interval data, App. Math. Comput., 175 (2006) 1375-1384.

[8] G. R. Jahanshahloo, F. Hosseinzadeh Lotfi, M. Izadikhah, Ranking DMUs with interval data using interval super efficiency index., Internathional Mathematical forum., 2 (2007) no, 9, 413-420.

[9] G. R. Jahanshahloo, F. Hosseinzadeh Lotfi, M. Moradi, Sensitivity and stability analysis in DEA with interval data, App. Math. Comput., 156 (2004) 463-477. 
[10] Y. K. Lee, K. S. Park, S. H. Kim, Identification of inefficiencies in additive model based IDEA, Computers and Operations Research, 29 (2002) 16611676.

[11] R. G. Thompson, L. N. Langemeier, C. T. Lee, E. Lee, R. M. Thrall, The role of multiplier bounds in efficiency analysis with applications to Kansas farming, J. Econometrics, 46 (1990) 93-108.

[12] J. Zhu, Imprecise data envelopment analysis(IDEA): A review and improvement with an application, European J. Oper. Res., 144 (2003) 513529.

\section{Received: November 17, 2006}

